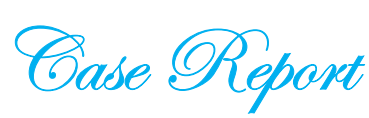

\section{Gower sign positive muscle weakness in a patient with short stature and hypophosphatemia}

\begin{abstract}
Omyma Sabir*, Abdelaziz Elamin*, Mohamed Elabyad*
\section{Abstract}

A 17-year-old Sudanese patient presented with short stature, generalized body aches, and proximal muscle weakness. He was seen by two orthopedic surgeons and an adult neurologist and misdiagnosed as having benign bone cysts, osteomalacia and Duchenne muscular dystrophy. In fact, he was suffering from severe hypophosphatemia, which manifest by unusual presentation. The diagnostic pitfalls are explained and the importance of urinalysis and measurement of bone minerals are emphasized.
\end{abstract}

Keywords: Renal tubular acidosis, Fanconi syndrome, Rickets.

$\mathrm{P}$ hosphorus is an inorganic anion. Most of the phosphorus in the human body is in the bone and the intracellular compartment. The plasma concentration of phosphorus is age dependent and does not reflect the total body stores because only $1 \%$ of phosphorus is found in the extracellular compartment. Children have higher concentrations of plasma phosphorus compared to adults because they have greater need for phosphorus to facilitate physical growth. Phosphorus as a component of ATP and other tri-nucleotides is critical for cellular energy metabolism. It is necessary for nucleic acid synthesis, and for formation of phospholipids, which is part of cell membranes and other structures. Along with calcium, phosphorus is an essential component of bone, and it is necessary for proper mineralization ${ }^{1-4}$. Through it is synergistic and reciprocal integration with calcium, phosphorus affects the action potential of skeletal muscles and its contractions. Hypophosphatemia is caused by tubular renal defects, hyperparathyroidism and nutritional factors ${ }^{5-9}$. When severe it manifest as rickets with hypotonia and muscle weakness ${ }^{10-11}$. Below we describe in details the clinical problem of a young patient with hypophosphatemia, who was misdiagnosed

*Gaffar Ibn Auf Children Teaching Hospital, Khartoum. Sudan.

Correspondence to Dr Elamin (azizmin@gmail.com ) and under treated because of unusual presentation.

\section{Case History:}

A previously healthy 17-year-old adolescent boy from Northern Sudan presented with difficulty in standing from sitting position for one year prior to admission, to Gaffer Ibn Auf Children Teaching Hospital in Khartoum. This condition was not associated with muscle or joint pain, morning stiffness, fever or skin rash. No post-exertional cramps or exercise intolerance. No symptoms related to the central or peripheral nervous system. It was not preceded by trauma and not affecting his walking so he did not seek medical advice at that time. Two months later he developed pain in his right ankle, moderate in severity, not associated with swelling or changes on the skin overlying the joint. It was neither related or affected by movement, nor associated or preceded by fever or trauma.

$\mathrm{He}$ presented first to the orthopedic department at the nearby regional hospital where he was investigated for his right ankle problem. The tests done include plain x-ray followed by a contrast-free axial and coronal $\mathrm{CT}$ of the right ankle. The films showed multiple lytic changes with sclerotic margins involving the distal metaphysis and epiphysis of the tibia and fibula. The report described presence of bone cyst and suggested infiltrative bone pathology (fig.1 and 2). 


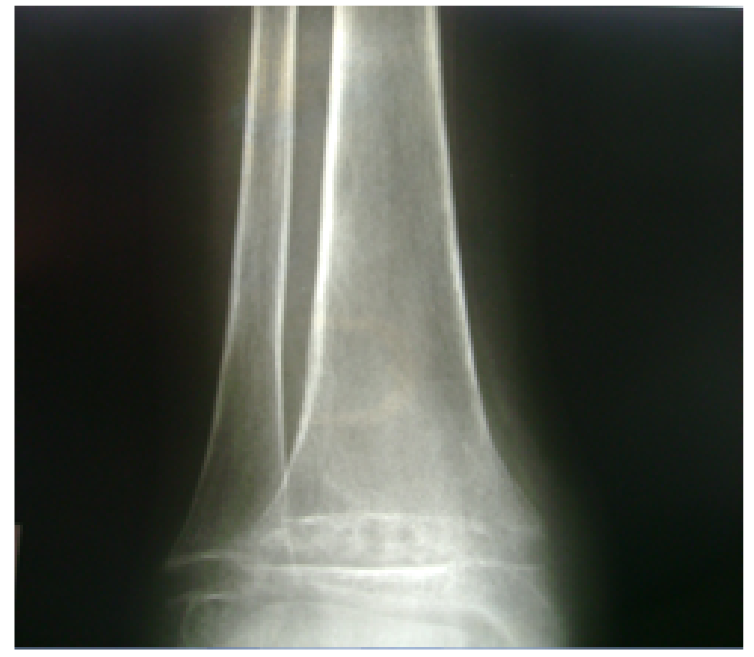

Fig.1: X-ray Rt. Ankle

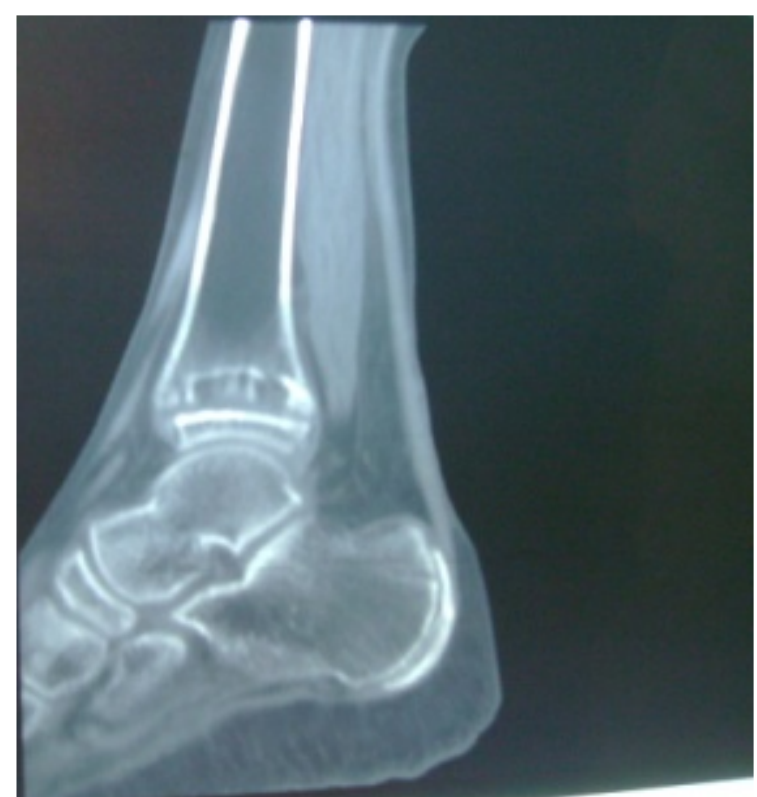

Fig.2:CT. Rt. Ankle

Afterwards a radio-isotope bone scan and bone biopsy were requested. Three phase bone scan was done with $333 \mathrm{MBq}$ of Tc-99m MDP and showed abnormal increased uptake of radioactivity in the blood vessels around the joints and delayed images of both feet suggestive of reflex sympathetic osteodystrophy. Also focal increased uptake was noted in both $8^{\text {th }}$ ribs and the right $6^{\text {th }}$ rib posteriorly (Fig 3 and 4).

The radiologist, in his report, attributed the focal lesions to trauma rather than infection, but he commented that metabolic bone diseases like osteomalacia and hyperparathyroidism needs to be excluded.

At that point, the surgeon decided to operate

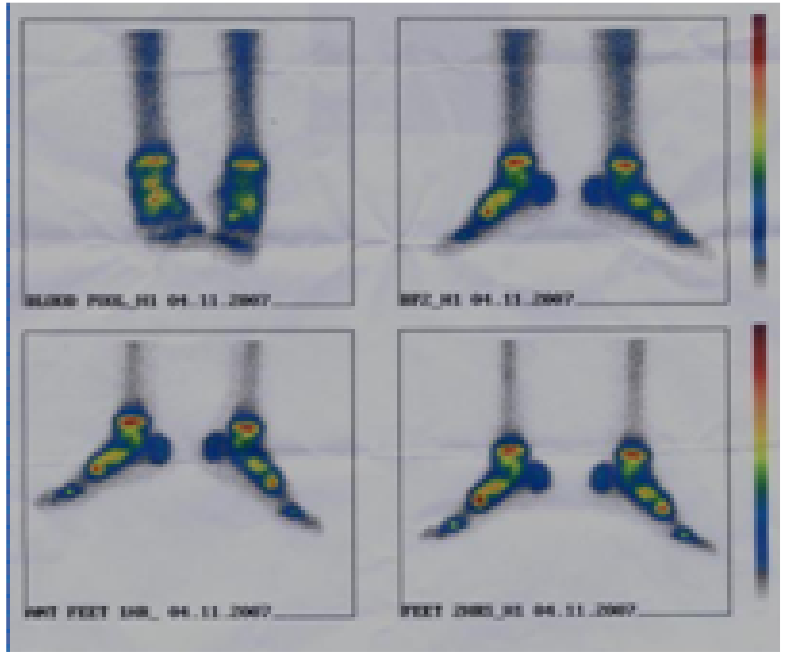

Fig 3.

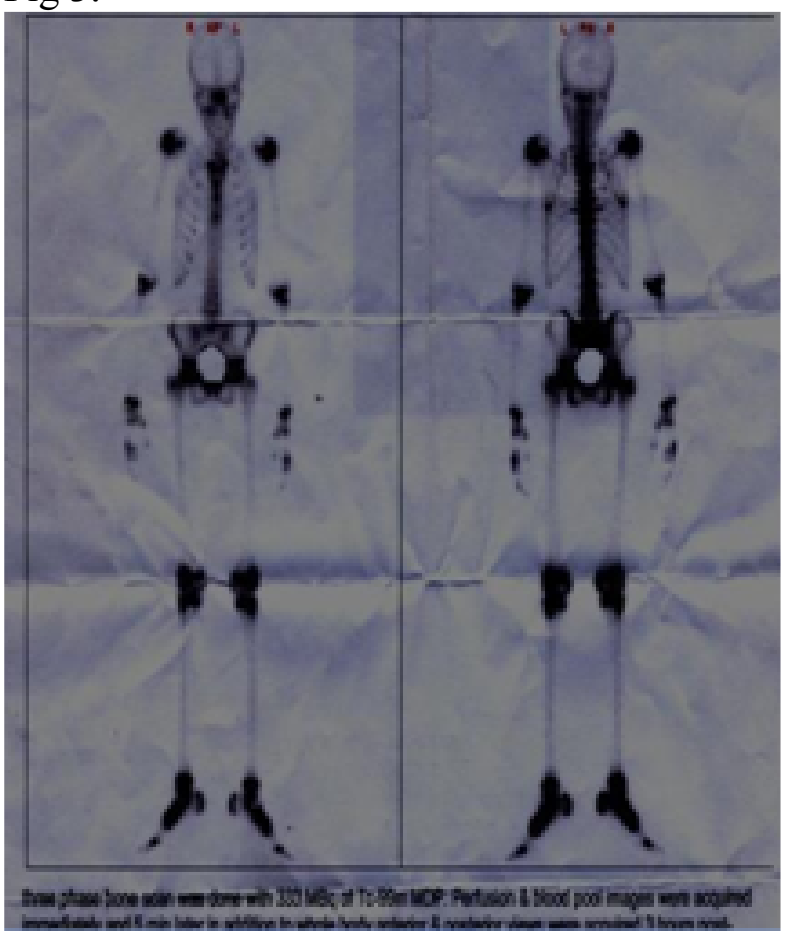

Fig 4.

Fig. 3 \& 4: Three phase bone scan with 333 MDPq of Tc-99m MDP.

on his right ankle. The bone cyst was excised and sent for histopathology. The lab report read benign bone degeneration with no evidence of malignancy or chronic inflammation. After the surgical operation the patient confined himself to bed for two days then he started to walk with support for ten days with difficulty in standing from sitting position. Thereafter, his condition became worse; he could not stand or walk or squat freely and he felt significant pain in both hips and knees with each effort done to move his 
lower limbs. However, his upper limbs were normal. He could lift his arms over his head and his handgrip was preserved. This time he consulted another orthopedic surgeon in a private clinic. He noted a positive Gower sign on examination. The child was then investigated and the tests results read as follows: $\mathrm{Hb} 13 \mathrm{~g} / \mathrm{dl}$, TWBC 9000 with normal differential, platelets 320, and ESR 62 $/ \mathrm{mm} / 1^{\text {st }}$ hour (westergren). Blood urea was $6.4 \mathrm{mmol} / 1$ (Ref. 2.5-7.5 mmol/1), plasma creatinine $53 \mathrm{mmol} / 1$ (Ref. $60-110 \mathrm{mmol} / \mathrm{l}$ ), serum sodium $134 \mathrm{mmol} / \mathrm{l}$, serum potassium $3.2 \mathrm{mmol} / \mathrm{l}$, serum phosphorus $0.44 \mathrm{mmol} / 1$ (Ref. 0.9-1.5 mmol/1), and serum calcium $2.08 \mathrm{mmol} / \mathrm{l}$ (Ref. 2.1-2.55 mmol/1). Liver function tests and enzymes were normal apart from a high serum level of ALP $1610 \mathrm{U} / \mathrm{L}$ (Ref. 65-260 U/L). A limited skeletal survey (only dorsal spine, pelvis, upper femoral, and chest X-ray films) was performed and showed decreased bone density. An EMG was done and suggested a myopathic pattern. The surgeon advised a muscle biopsy, which was taken from the right quadriceps femoris muscle. The section of the muscle biopsy showed thin muscle fibers with aggregate nuclei, and without remarkable inflammation. The histological features were reported as consistent with a diagnosis of muscular dystrophy. The treating doctor diagnosed the child as having Duchenne muscular dystrophy based upon the positive Gower sign, the EMG result and the muscle biopsy report despite normal level of serum Creatinine Kinase and absence of pseudohypertrophy of calf muscles. Parents were told that no treatment is available for his problem and advised physiotherapy. They were not convinced and sought medical advice at a neurology clinic. Without doing any further investigations, the physician prescribed calcium tablets and advised the patient to expose himself to sunlight. The condition deteriorated with the calcium therapy, so the parents took the patient to a pediatrician for the first time. The doctor examined the child, did few simple tests and referred the child to our unit at Gaffar Ibn Auf Children Teaching Hospital for further evaluation and management.
We interrogated the patient and noted the following history:

- Parents are first degree cousins; mother is hypertensive on Atenolol and no history of hereditary diseases in the family.

- He is the shortest pupil since he joined the school at age 7 yrs and has poor appetite and poor weight gain since he was thirteen years old. Constipation for the last five months but no vomiting, abdominal pain or distension.

- Polyuria and nocturia for the last four months but no enuresis, dysuria or urgency.

- No past history of renal problems and he was not on regular medication. He had malaria, which was treated by Artemether injections.

Examination showed no dysmorphic features and normal vital signs. Both his weight and BMI were below the 3rd percentile for age and sex and his height was -4 SD below the mean. The body's upper over lower segment ratio was 1.0, and arm span approximate his height. His pubertal development equals Tanner stage 4. Hand, head, neck and ENT examination were normal. Physical eye examination was normal. Slit lamp examination did not reveal Kayser-Fleischer rings, cystine crystals or iritis. Fundoscopy revealed normal, fundi, no maculopathy, retinitis pigmentosa or signs of retinopathy. Neurologic and musculoskeletal examination found symmetrical muscle wasting more in the lower limbs than the upper limbs, and affecting the proximal muscles more than the distal muscles Muscle weakness was evident and Gower sign was positive. The power in the small muscles of both hands and forearms was normal but grade 4 in the pectorals, supraspinatus and the deltoid muscles on both sides of the body. A mild kyphosis in his thoracic spine was also noted. Power in the muscles of the toes and ankles was normal bilaterally, but grade 2 in the extensors \& flexors of both knees and grade 2 in the extensors, flexors, adductors and abductors of 
the hip bilaterally. There was pain while actively moving the hips and knees bilaterally. No joints stiffness, swelling, tenderness, deformity or skin changes (apart of two scars of operation of what assumed to be bone cyst in his right ankle and scar of muscle biopsy in his right thigh). No bowing of legs, pseudo-hypertrophy of calves muscles, genu varum or coax vara.

Investigations showed: normal FBC, urine analysis showed acidic reaction ( $\mathrm{PH} 6.5$ ), specific gravity 1005 (Ref. 1020-1030), glucosuria $(4+)$ and proteinuria $(1+)$. Blood glucose fasting \& 2 hours after meal were normal. The glomerular filtration rate, blood urea, serum creatinine, and electrolytes were normal. Serum calcium was normal and serum phosphorus was very low (0.09 $\mathrm{mmol} / \mathrm{l})$. Serum alkaline phosphatase was high $(1610 \mathrm{u} / \mathrm{l})$ and Creatinine Kinase was normal $17 \mathrm{iu} / 1$ (Ref. 24-190 iu/l). Blood gas analysis showed hyperchloremic metabolic acidosis with normal anion gap. Parathyroid hormone was normal and tests for connective tissue diseases were negative. Urine for metabolic screening test was negative and urinary electrolytes were normal apart from hyperphosphaturia. Vitamin D metabolites were normal.

The x-ray film of the left wrist showed decreased bone density with fraying in the distal ends of the ulna and the radius and bone age between 12 and 13 years. The thoracic spine x-ray film showed also decreased bone density with increased anterioposterior curvature (fig. 5 and 6).

Abdominal sonography showed small kidneys $7.5 \mathrm{~cm}$ each with poor medullo-cortical differentiations indicating chronic renal paranchymal disease. There were no obstructive changes, calculi, cyst or nephrocalcinosis. Renal biopsy was taken and showed no evidence of glomerular, tubular or interstitial lesions.

Based on the history, the clinical examination, the biochemical and the radiological features; we made a diagnosis of hypophosphatemia secondary to proximal renal tubular acidosis, which is due to Fanconi syndrome.

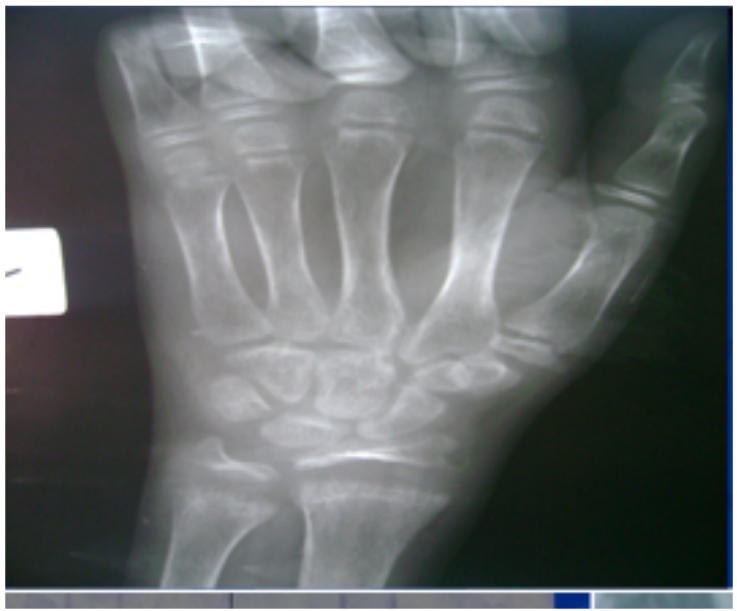

Fig 5. X- ray Lt. Wrist

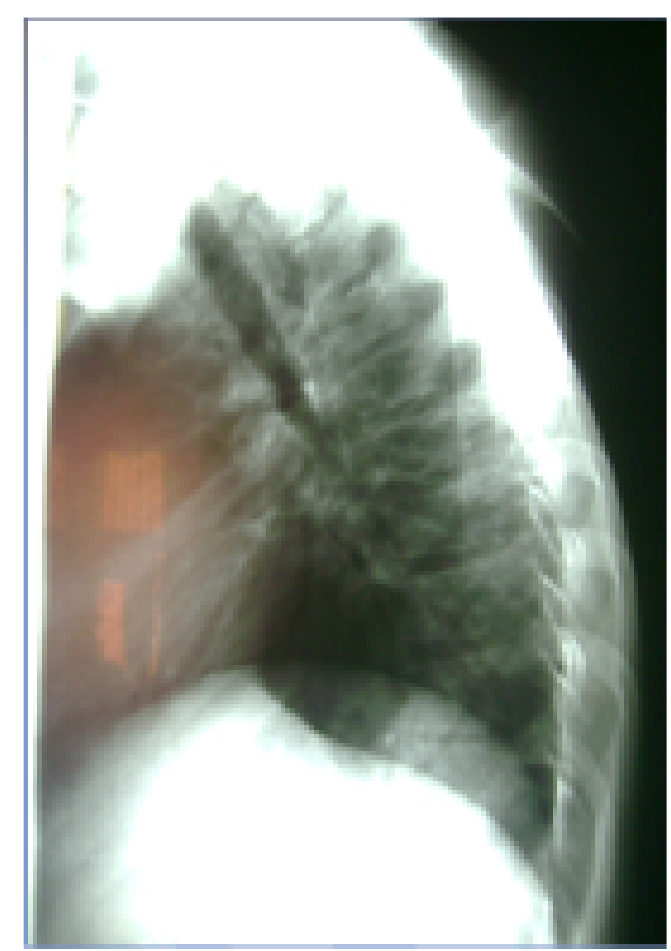

Fig 6. X-ray thoracic spine, lateral view

We prescribed the following medications: sodium bicarbonate $500 \mathrm{mg} 6$ hourly, potassium syrup $20 \mathrm{ml} 6$ hourly, phosphate tablet $500 \mathrm{mg} 6$ hourly, and alphacalcidol tablet 1 microgram OD. Physiotherapy was to be initiated when bone lesions healed. One month after starting therapy, the biochemical abnormalities returned to normal including the plasma phosphorus level and metabolic acidosis. The patient's overall quality of life improved substantially; he was able to stand and walk without problems and he returned to school after the long sick-leave. 


\section{Pitfalls of Diagnosis:}

This patient presented with difficulty in standing from sitting position, which suggests proximal muscle weakness, together with bone pain in his lower limbs. However, the attending doctor, an orthopedic surgeon, ignored the muscle weakness and concentrated on the pain around the right ankle joint. He ordered bone x-ray film, bone $\mathrm{CT}$, and radio-isotope bone scans. The bone scan report was suggestive of reflex sympathetic osteodystrophy and advised exclusion of metabolic bone diseases. The doctor in charge ignored that report too and with full trust in the radiological report, which claimed presence of bone cyst and infiltrative bone pathology, he proceeded to surgical exploration and open bone biopsy that proved to be normal. We showed the x-ray films to two experienced radiologist in Khartoum and both reported (independently) that there was marked decreased in the bone density and what assumed to be bone cysts in the distal part of the tibia and fibula, was just haziness and irregularity at the zone of ossification center of these bones, which is a known feature of early rickets.

In his second consultation, the patient was investigated again by an orthopedic surgeon. The lab investigations showed marked hypophosphatemia, hypokalemia, high alkaline phosphatase, and the skeletal survey reported markedly decreased bone density. Nevertheless, a muscle biopsy was taken and diagnosis of Duchenne muscular dystrophy made based mainly on EMG and the muscle biopsy report. This diagnosis was not farfetched, because the patient is a male and has history of progressive proximal muscle weakness with positive Gower sign. What makes it unlikely were the negative family history and absence of characteristic features of DMD (pseudohypertrophy of calves muscles, macroglossia, cardiopulmonary involvement, and absent deep tendon reflexes in the upper extremities and patella). Also the patient has features that can't be explained by DMD (polyuria \& polydipsia, short stature and the biochemical abnormality). In addition, the histological features described in the biopsy report don't support the diagnosis of DMD, which is characterized by variations in muscle fiber sizes with focal areas of degenerating \& regenerating fibers.

In his third consultation, the patient was reviewed by a neurologist who prescribed calcium and advised exposure to sunlight. The contrast between the surgeon \& the physician approach is clear here. By reviewing the previous lab tests, the physician made the correct diagnosis of rickets, but he failed to recognize its type or course; simply because he didn't do further tests. This mistake was avoided by the pediatrician who asked for urinalysis, which revealed the glucosuria and the proteinuria.

When we evaluated the patient, we noted the relevant history and physical signs and did thorough investigations. We documented the metabolic acidosis, the hypokalemia and hypophosphatemia, aminoaciduria and glucosuria with normal blood glucose concentration and did a renal biopsy. We made the diagnosis of hypophosphatemic rickets secondary to Fanconi syndrome and prescribed treatment, but that is not the end of the road. Fanconi syndrome has many causes (table 1), and further tests are needed to determine the exact type.

Table 1: Causes of Fanconi Syndrome

\begin{tabular}{l|l} 
Category & Disease \\
\hline Sporadic & Idiopathic \\
\hline Genetic & Cystinosis \\
& Lowe syndrome \\
& Galactosemia/ Tyrosinemia \\
& Hereditary fructose intolerance \\
& Wilson disease \\
& Dent disease \\
& Fanconi-Bickel syndrome \\
& Mitochondrial disease \\
\hline Acquired & Heavy metal poisoning \\
& Sodium Valproate \\
& Gentamycin \\
& Outdated Tetracycline \\
& Cyclosporine \\
& Ifosfamide \\
& Vitamin D deficiency
\end{tabular}


This is important for genetic counseling and for academic reasons, but from management point of view all types are treated the same way.

\section{Discussion:}

The genetic and hereditary causes of Fanconi syndrome are illustrated in Table 1. The commonest inherited cause is Cystinosis, a systemic disease caused by a defect in the metabolism of the aminoacid cystine ${ }^{12-15}$. It is characterized by accumulation of cystine crystals in many organs notably the kidneys and the eyes. Patients usually present early, have fair skin and liver involvement. Slit lamp examination of the eye and renal biopsy confirm the diagnosis. Both were negative in our patient. The clinical findings together with the negative family history confidently exclude cystinosis and the other hereditary conditions (galactosemia, tyrosinemia, Lowe syndrome, Fanconi-Bickel syndrome, Wilson disease and Dent disease) ${ }^{16-19}$ as possible causes of Fanconi syndrome in our patient. Although the idiopathic sporadic type of Fanconi syndrome is rare and occurs mainly in Caucasians, it remains the most likely cause of the condition in our patient. It has been reported before in blacks, Hispanics, and people of Middle Eastern descent ${ }^{20-21 .}$

The acquired causes of Fanconi syndrome are also shown in Table 1 above. It includes heavy metal poisoning, drug toxicity and vitamin $\mathrm{D}$ deficiency ${ }^{22}$. Of note is the association between the commonly used anticonvulsant medication, Sodium Valproate, and renal tubular acidosis 23-26. Another relatively new drug, Ifosfamide, used in the treatment of Wilm's tumour and some other solid tumors in children is emerging as a potent induced of renal tubular acidosis ${ }^{27-28}$. All the known secondary causes of Fanconi syndrome were excluded in our patient. Serum levels of vitamin D metabolites (25 hydroxycalciferol and 1, 25 dihydroxycholecalciferol) were normal and there was no history of heavy metals or drugs ingestion. The only medicine the patient received was Artemether injections for falciparum malaria two months before his current illness. Artemether is a herbal-derived Chinese medicine not known to cause secondary Fanconi syndrome. However, nephropathy has been reported following ingestion of another herbal Chinese medicine, which was used to treat obesity in a woman from Belgium ${ }^{29}$. Also a popular Chinese herbal remedy containing Arsitolochic acid, which is used to treat muscular and body aches in China, Korea and Japan, has been identified to cause revisable transient Fanconi syndrome ${ }^{30}$. The pharmaco-kinetic studies on Artemether compounds proved that sodium Artesunate inhibits sodium chloride transport in the thick ascending limb of the loop of Henle and therefore has a diuretic and natriuretic effect. But no evidence to date that Artemether causes proximal renal tubular acidosis.

\section{Conclusion:}

The case highlights the relationship between hypophosphatemia and proximal myopathy. It also emphasizes the importance of urinalysis and measurement of bone minerals in all patients with unusual, persistent musculoskeletal symptoms. The association of Artemether and Fanconi needs further exploration.

\section{Acknowledgments}

We are very grateful to Dr. Mohamed Babiker Abdulrahim, Assistant professor of Child Health, university of Khartoum \& consultant pediatric nephrologist at Soba university hospital (SUH) who did the renal biopsy. We also thank the staff of the labs at SUH \& Elneileen Medical Centre for doing some of the tests. 


\section{References:}

1. Gattineni J, Baum M. Regulation of phosphate transport by fibroblast growth factor 23 (FGF23): implications for disorders of phosphate metabolism. Pediatr Nephrol 2009; 133: 876-881.

2. Bastepe M, Jappner H. Inherited hypophosphatemic disorders in children and the evolving mechanisms of phosphate regulation. Rev Endocrinol Metab Disord 2008; 9(2): 171-180.

3. Shaikh A, Berndt T, Kumar R. Regulation of phosphate homeostasis by the phosphatonins and other novel mediators. Pediatr Nephrol 2008; 23(8): 12031210.

4. Skowroa R, Jawiak E, Lorenc RS. Metabolic bone disease in children: etiology and treatment options. Treat Endocrinol 2006; 5(5): 297-318.

5. Ward LM. Renal phosphate--wasting disorders in childhood. Pediatr Endocrinol Rev 2005; 2 (Suppl 3): 342-350.

6. Vaisbich MH, Koch VH. Hypophosphatemic rickets: results of a long-term follow-up. Pediatr Nephrol 2006; 21(2): 230-234.

7. Laroche M, Boyer JF. Phosphate diabetes, tubular phosphate reabsorption and phosphatonins. Joint Bone Spine 2005; 72(5): 376-381.

8. Cho HY, Lee BH, Kang JH, et al. A clinical and molecular genetic study of hypophosphatemic rickets in children. Pediatr Res. 2005; 58(2): 329-333.

9. Gribaa M, Younes M, Bouyacoub Y, et al. An autosomal dominant hypophosphatemic rickets phenotype in a Tunisian family caused by a new FGF23 missense mutation. J Bone Miner Metab 2009; 78: 644-649.

10. Sochett E, Doria AS, Henriques F, et al. Growth and metabolic control during puberty in girls with $\mathrm{X}$ linked hypophosphataemic rickets. Horm Res 2004; 61(5): 252-256.

11. Esterberg J, Kassim RA, Redmon J et al. Hip pain in a case of hypophosphatemic osteomalacia. Am J Orthop 2003; 32(9): 455-458.

12. Wornell P, Crocker J, Wade A et al. An Acadian variant of Fanconi syndrome. Pediatr Nephrol 2007; 22(10): 1711-1715.

13. Nesterova G, Gahl W. Nephropathic cystinosis: late complications of a multisystem disease. Pediatr Nephrol 2008; 23(6): 863-878.

14. Servais A, Moriniare V, Granfeld JP, et al. Lateonset nephropathic cystinosis: clinical presentation, outcome, and genotyping. Clin J Am Soc Nephrol 2008; 3(1): 27-35.

15. Santer R, Steinmann B, Schaub J. Fanconi-Bickel syndrome--a congenital defect of facilitative glucose transport. Curr Mol Med 2002; 2(2): 213-227.

16. Bayrakci US, Baskin E, Ozcay F et al. Renal Fanconi syndrome and myopathy after liver transplantation: drug-related mitochondrial cytopathy? Pediatr Transplant 2008; 12(1): 109-112.

17. Au KM, Lau SC, Mak YF, et al. Mitochondrial DNA deletion in a girl with Fanconi's syndrome. Pediatr Nephrol 2007; 22(1): 136-140.

18. Cobeaas CJ, Rahman RC. A 3-year-old child with proteinuria and nephrocalcinosis. Suspicion of Dent disease. Pediatr Nephrol 2004; 19(11): 1300-1302.

19. Cheong HI, Lee JW, Zheng SH, et al. Phenotype and genotype of Dent's disease in three Korean boys. Pediatr Nephrol 2005; 20(4): 455-459.

20. Hsu SY, Tsai IJ, Tsau YK. Comparison of growth in primary Fanconi syndrome and proximal renal tubular acidosis. Pediatr Nephrol 2005; 20(4): 460-464. 21. Bagga A, Bajpai A, Menon S. Sporadic primary renal tubular disorders. Indian J Pediatr 2005; 72(9): 771-716.

22. Taylor HC, Elbadawy EH. Renal tubular acidosis type 2 with Fanconi's syndrome, osteomalacia, osteoporosis, and secondary hyperaldosteronism in an adult consequent to vitamin $\mathrm{D}$ and calcium deficiency: effect of vitamin D and calcium citrate therapy. Endocr Pract 2006; 12(5): 559-567.

23. Watanabe T, Yoshikawa H, Yamazaki $\mathrm{S}$ et al. Secondary renal Fanconi syndrome caused by valproate therapy. Pediatr Nephrol 2005; 20(6): 814817.

24. Watanabe T, Nakayasu K, Nagayama Y. Autoimmune hemolytic anemia and renal Fanconi syndrome caused by valproate therapy. Eur J Pediatr 2005; 164(3): 186-187.

25. Yoshikawa H, Watanabe $T$, Abe $T$. Fanconi syndrome caused by sodium valproate: report of three severely disabled children. Eur J Paediatr Neurol 2002; 6(3): 165-167.

26. Knorr M, Schaper J, Harjes M et al. Fanconi syndrome caused by antiepileptic therapy with valproic Acid. Epilepsia 2004; 45(7): 868-871.

27. Church DN, Hassan AB, Harper SJ et al. Osteomalacia as a late metabolic complication of Ifosfamide chemotherapy in young adults: illustrative cases and review of the literature. Sarcoma 2007; 27: 91586.

28. Skinner R. Chronic ifosfamide nephrotoxicity in children. Med Pediatr Oncol 2003; 41(3): 190-197.

29. Nissenkorn A, Korman SH, Vardi O, et al. Fanconi syndrome following ingestion of Chinese herbal medicine in a Belgian lady of Moroccan origin. J Nephrol 2001; 16(9): 642-644.

30. Hong YT, Fu LS, Chung LH et al. Fanconi's syndrome, interstitial fibrosis and renal failure by aristolochic acid in Chinese herbs. Pediatr Nephrol 2006; 21(4): 577-579. 